\title{
Disruption of O-GIcNAc cycling in C. elegans perturbs nucleotide sugar pools and complex glycans
}

\section{Salil K. Ghosh ${ }^{1 \dagger}$, Michelle R. Bond ${ }^{1}$, Dona C. Love ${ }^{1}$, G. Gilbert Ashwell ${ }^{1 \neq}$, Michael W. Krause ${ }^{2}$ and John A. Hanover ${ }^{1 *}$}

${ }^{1}$ Laboratory of Cell and Molecular Biology, National Institute of Diabetes and Digestive and Kidney Diseases, National Institutes of Health, Bethesda, MD, USA

${ }^{2}$ Laboratory of Molecular Biology, National Institute of Diabetes and Digestive and Kidney Diseases, National Institutes of Health, Bethesda, MD, USA

\section{Edited by:}

Tony Lefebvre, University Lille 1, France

Reviewed by:

Gerald W. Hart, Johns Hopkins Medical School, USA

Lance Wells, University of Georgia, USA

\section{*Correspondence:}

John A. Hanover, Laboratory of Cell and Molecular Biology, National Institute of Diabetes and Digestive and Kidney Diseases, National Institutes of Health, Building 8 Room B127, 9000 Rockville Pike, Bethesda, MD 20892, USA

e-mail: jah@helix.nih.gov

${ }^{+}$Present address:

Salil K. Ghosh, Food and Drug Administration, Center for Biologics Evaluation and Research, Silver Spring, MD, USA

${ }^{\ddagger}$ Deceased.
The carbohydrate modification of serine and threonine residues with O-linked beta$\mathrm{N}$-acetylglucosamine (O-GlcNAc) is ubiquitous and governs cellular processes ranging from cell signaling to apoptosis. The O-GIcNAc modification along with other carbohydrate modifications, including N-linked and O-linked glycans, glycolipids, and sugar polymers, all require the use of the nucleotide sugar UDP-GICNAc, the end product of the hexosamine biosynthetic pathway (HBP). In this paper, we describe the biochemical consequences resulting from perturbation of the O-GIcNAc pathway in $C$. elegans lacking O-GIcNAc transferase and O-GIcNAcase activities. In ogt-1 null animals, steady-state levels of UDPGIcNAc/UDP-GalNAc and UDP-glucose were substantially elevated. Transcripts of genes encoding for key members in the HBP (gfat-2, gna-2, C36A4.4) and trehalose metabolism (tre-1, tre-2, tps-2) were elevated in ogt-1 null animals. While there is no evidence to suggest changes in the profile of N-linked glycans in the ogt-1 and oga-1 mutants, glycans insensitive to PNGase digestion (including O-linked glycans, glycolipids, and glycopolymers) were altered in these strains. Our data support that changes in O-GlcNAcylation alters nucleotide sugar production, overall glycan composition, and transcription of genes encoding glycan processing enzymes. These data along with our previous findings that disruption in O-GlcNAc cycling alters macronutrient storage underscores the noteworthy influence this posttranslational modification plays in nutrient sensing.

Keywords: O-GIcNAcylation, nucleotide sugars, hexosamines, $C$. elegans/nematode, glycogen, trehalose

\section{INTRODUCTION}

Posttranslational modifications ranging from glycosylation to phosphorylation play critical roles in biological processes including protein localization, transcription, and cellular signaling [see Ref. (1) and references therein]. The posttranslational modification O-linked beta- $N$-acetyl glucosamine (O-GlcNAc) is ubiquitous throughout the nucleus and cytoplasm modifying over 4000 protein substrates including nuclear pore and transcription complexes, proteasomes, and kinases (2). The addition and removal of O-GlcNAc to serine and threonine residues is governed by two enzymes, O-GlcNAc transferase (OGT) and O-GlcNAcase (OGA), respectively (3). This dynamic cycling occurs faster than protein turnover poising O-GlcNAc to act as a signaling molecule (4).

UDP-GlcNAc, the activated nucleotide sugar utilized by OGT, is the end product of the hexosamine biosynthetic pathway (HBP). A metabolite responsible for sensing the nutrient status of the cell, UDP-GlcNAc is synthesized by a series of enzymes utilizing key metabolites including glucose, L-glutamine, acetyl-CoA, and UTP (5). Perturbation of the HBP is linked with modulation in insulin signaling and glucose toxicity [see Ref. (6) and references therein]. Indeed, globally decreased levels of UDP-GlcNAc can be profoundly damaging to mammals (7). The way in which the HBP plays a role in the modulation of insulin signaling and glucose toxicity is currently being defined with implications that
O-GlcNAcylation plays an important mechanistic role [see Ref. (8) and references therein].

We previously demonstrated that C. elegans loss-of-function ogt-1 and oga-1 animals have altered insulin signaling and carbohydrate-fat metabolism (9). Importantly, ogt-1 and oga-1 null C. elegans are viable while loss of OGT and OGA activity in higher eukaryotes yields embryonic lethality $(10,11)$. C. elegans lacking OGT-1 activity exhibit no addition of O-GlcNAc to protein serine and threonine residues and animals without OGA-1 activity lack the capacity to remove the modification. In order to better understand the repercussions of impaired O-GlcNAc cycling in a whole organism, we chose to define the way in which these perturbations altered UDP-GlcNAc concentrations, glycan composition, and transcription of important metabolic enzymes. Among the most striking differences, we found that animals lacking OGT-1 or OGA-1 activities exhibit increases in UDP-HexNAc pools and differences in their overall glycan compositions compared to wild type (N2). Indeed, along with these changes, ogt-1 null animals also show increased transcription of metabolic and HBP genes suggesting that the animals are modulating or attempting to compensate for the altered carbohydrate profiles. Although O-GlcNAc cycling has been linked to nutrient sensing, we provide the first evidence in a whole organism that O-GlcNAc plays an important role in modulating nucleotide sugar utilization and the steady-state 
levels of transcripts encoding key HBP enzymes. We suggest that $\mathrm{O}-\mathrm{GlcNAc}$ acts as a rheostat to fine-tune evolutionarily conserved components of $C$. elegans metabolism giving a defined model to study how changes in O-GlcNAc may impact human health.

\section{MATERIALS AND METHODS}

\section{ELEGANS AND BACTERIAL STRAINS AND MAINTENANCE}

The following C. elegans strains were used in this study: N2 Bristol (WT), ogt-1 (ok430), ogt-1 (tm1046), and oga-1 (ok1207). The ogt-1 (ok430) and oga-1 (ok1207) strains were provided by the C. elegans Gene Knockout Consortium (CGC, Oklahoma Medical Research Foundation, Oklahoma City) and the ogt-1 (tm1046) was provided by the National Bioresource Project of Japan. All strains were backcrossed four times to N2 prior to use in experiments. The presence of the deletion alleles was confirmed by nested PCR primers as described by the CGC. The OP50 E. coli was cultured without antibiotic at room temperature in Luria-Bertani (LB) broth and plated on nematode growth media (NGM) plates or $2 \%$ agarose-topped LB plates. NGM plates were made with tryptone rather than peptone. C. elegans were maintained on NGM plates supplemented with OP50 E. coli and animals were manipulated using standard techniques $(12,13)$. Two percent agarose-topped LB plates were used for RNA isolation experiments only.

\section{ISOLATION OF NUCLEOTIDE SUGAR}

C. elegans strains were grown in large quantities starting from synchronous L1 stage and collected when animals reached the gravid adult stage (after approximately $72 \mathrm{~h}$ incubation at room temperature, $22^{\circ} \mathrm{C}$ ). Animals were washed from NGM plates with water, washed twice with water, counted, and purged by rocking animals for $30 \mathrm{~min}$ in water at room temperature as previously described (9). Animals were isolated by centrifugation and the supernatant removed first by pipette and then by lyophilization. To $8 \mathrm{mg}$ lyophilized worms, $0.75 \mathrm{ml}$ cold $0.5 \mathrm{~N}$ perchloric acid was added. The suspension was vortexed vigorously for $20 \mathrm{~s}$ in an ice bath followed by centrifugation at $15,000 \times g$ for $10 \mathrm{~min}$ at $4^{\circ} \mathrm{C}$ and the supernatant was collected. The remaining pellet was extracted second time and the two supernatants was pooled. Two hundred microliters of charcoal suspension $(30 \mathrm{mg}$ of Mallinckrodt charcoal/ml of $1 \mathrm{~N}$ perchloric acid) was added to the cold supernatant and stirred vigorously in an ice bath. The activated charcoal in acidic solution binds the nucleotide sugar and the sugars are then released in the alkaline solution in the following steps. After centrifugation at $15,000 \times g$ for $10 \mathrm{~min}$ at $4^{\circ} \mathrm{C}$, the supernatant was discarded. The charcoal pellet was eluted three times with $750 \mu \mathrm{L}$ of a solution containing $50 \%$ ethanol $+1 \% \mathrm{NH}_{4} \mathrm{OH}$. The EtOH solutions were isolated, pooled, frozen, and lyophilized. This experiment was performed in triplicate. Isolated sugars were analyzed by high performance anion exchange chromatography (HPAEC) with pulse amperometric detector (PAD) using a PA10 anion exchange column as described by Suriano et al. (14). Error bars represent SD of an experiment done in triplicate and $P$ values were calculated by an unpaired Student's $t$-test. ns: $P>0.05$; ${ }^{\star} P<0.05$ compared to N2.

\section{ISOLATION OF PNGaSe-SENSITIVE GLYCANS}

Glycoprotein rich fractions were isolated from C. elegans at synchronous L4 larval stage by procedures described by Cipollo et al. $(15,16)$. Further processing was analogous to preparation described in the aforementioned papers with minor modifications. Isolated glycoproteins were treated with L-1-tosylamido2-phenylethyl chloromethyl ketone trypsin for $4 \mathrm{~h}$ at $37^{\circ} \mathrm{C}$ in $50 \mathrm{mM}$ ammonium bicarbonate, $\mathrm{pH} 8.5$ buffer. The reaction was stopped by boiling the samples twice for $10 \mathrm{~min}$. The resulting peptides were isolated by acetone $(80 \%)$ precipitation. $\mathrm{N}$ glycans were released from these glycopeptides by incubating with PNGase A (Roche) and F (Prozyme) enzymes according to the manufacturers' protocols. PNGase-sensitive glycans were pooled and purified by passing through LudgerClean ${ }^{\mathrm{TM}}$ EB10 Glycan Cleanup Cartridge following the manufacturer's protocol. Resulting glycans were hydrolyzed with $2 \mathrm{M}$ TFA for $4 \mathrm{~h}$ at $100^{\circ} \mathrm{C}$ to yield monosaccharides. Samples were evaporated to dryness and resuspended in $100 \mu \mathrm{L}$ of deionized water. This evaporation and resuspension was repeated twice with the last $100 \mu \mathrm{L}$ water suspension passed through a cation exchange resin to remove amino acids and peptides. A portion of this resulting monosaccharidecontaining solution was profiled by HPAEC-PAD as described elsewhere $(14,17)$. To measure GalNAc and GlcNAc, the concentrations of monosaccharides galactosamaine (GalN) and glucosamine $(\mathrm{GlcN})$ were measured by HPAEC as, immediately following their release, the $N$-acetyl sugars undergo quantitative deacetylation. Error bars represent SD of an experiment done in triplicate, and $P$ values were calculated by an unpaired Student's $t$-test. ns: $P>0.05 ;{ }^{\star} P<0.05 ;{ }^{\star \star} P<0.005 ;{ }^{\star * \star} P<0.0005$ compared to N2.

\section{ISOLATION OF RNA FROM L4 STAGE C. ELEGANS}

OP50 grown on agarose coated LB plates were seeded with synchronized L1 N2, ogt-1 (ok430), and oga-1 (ok1207) strains. Animals incubated at room temperature $\left(22^{\circ} \mathrm{C}\right)$ were collected after roughly $48 \mathrm{~h}$ when $\mathrm{N} 2$ animals reached a synchronous L4 stage. Although animals were synchronous at L1, we noticed that ogt-1 (ok430) animals had a difference in growth rate and we accommodated by plating them on OP50 $4 \mathrm{~h}$ prior to the other strains. Collected L4 populations contained 97, 95, 80, and 60\% L4 animals in N2, oga-1, ogt-1 (tm1046), and ogt-1 (ok430) strains, respectively. The remaining animals ranged from stages L1 to L3. C. elegans were isolated from plates, thoroughly washed with water to remove bacteria, and worm pellets were stored $-80^{\circ} \mathrm{C}$ for further use. RNA was isolated from each strain using a Qiagen RNAeasy Mini kit and quantified spectrophotometrically as described in Ref. $(4,18)$.

\section{qRT-PCR ANALYSIS}

RNA was treated with DNAse from Invitrogen to destroy contaminating genomic DNA in RNA sample $(4,18)$. SuperScript III (Invitrogen) was used to produce cDNA according to manufacturer's protocols with random primer from Promega. Following cDNA synthesis, quantitative real-time PCR (qRT-PCR) was performed using SYBR Green to quantitatively determine gene expression. RNA treated without reverse transcriptase served as the negative control. The experiment was performed in biological triplicate and samples were normalized to the control gene act-4. qRT-PCR data were analyzed by the comparative $C_{\mathrm{t}}$ method, error bars represent SD, and $P$ values were calculated by 


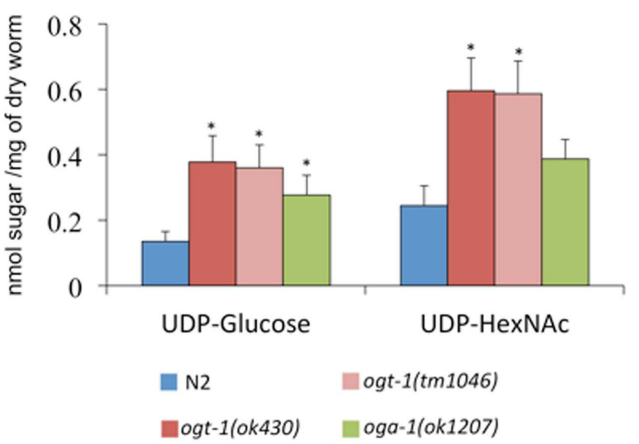

FIGURE 1 | O-GIcNAc cycling mutants exhibit increased concentrations of nucleotide sugar. UDP-nucleotide sugars were assessed by HPAEC-PAD detection. Compared to N2 animals, ogt-1 (ok430), ogt-1 (tm 1046), and oga-1 (ok1207) animals have increased levels of UDP-nucleotide sugar. Error bars represent SD of an experiment done in triplicate. ns: $P>0.05 ;{ }^{*} P<0.05$ compared to N2.

an unpaired Student's $t$-test. ns: $P>0.05 ;{ }^{\star} P<0.05 ;{ }^{\star \star} P<0.005$; ${ }^{* *} P<0.0005$ compared to N2.

\section{GENE ACCESSION INFORMATION}

Gene public name, Gene WormBase ID: ogt-1, K04G7.3, WBGene 00003858; oga-1, T20B5.3, WBGene00020596; gfat-2, F22B3.4, WBGene00009035; gna-1, B0024.12, WBGene00001646; gna-2, T23G11.2, WBGene00001647; C36A4.4, WBGene00007965; tre-1, F57B10.7, WBGene00006607; tps-2, F19H8.1, WBGene00006603; gsy-1, Y46G5A.31, WBGene00001793; Y73B6BL.4, WBGene00022 233; act-4, M03F4.2, WBGene00000066.

\section{RESULTS}

\section{NUCLEOTIDE SUGAR LEVELS ARE INCREASED IN C. ELEGANS ogt-1 AND oga-1 MUTANTS}

The C. elegans nucleocytoplasmic enzyme OGT-1 relies on a portion of the UDP-GlcNAc pool to glycosylate serine and threonine residues with O-GlcNAc. We sought to determine the upstream consequences when either OGT-1 activity, or the activity of its counterpart OGA-1, were absent in C. elegans. We initially profiled activated nucleotide sugar concentrations by isolating the nucleotide sugar pools, treating them with mild acid hydrolysis, and profiling the freed monosaccharides by HPAEC-PAD (Figure 1). We were intrigued to find that animals lacking either OGT-1 or OGA-1 activity demonstrated increased pools of UDPglucose and ogt-1 animals had increased UDP-GlcNAc/UDPGalNAc (represented as UDP-HexNAc) pools. Loss of OGT-1 yielded animals with over a twofold increase in these nucleotide sugars compared to $\mathrm{N} 2$ while oga-1 animals demonstrated a more modest increase. Importantly, the increased nucleotide sugar pool was similarly elevated in both $C$. elegans ogt-1 null alleles that we used throughout the paper to further support our conclusions (Figure 1). Additional nucleotide sugars profiled (GDP-mannose, GDP-fucose, and UDP-galactose) were present in minimal amounts and their concentrations remained unchanged in ogt-1 (ok430), ogt-1 (tm1046), and oga-1 (ok1207) animals compared to N2 (data not shown).

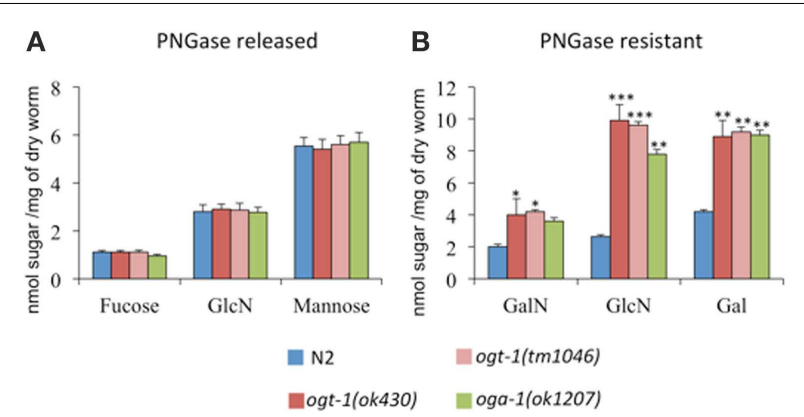

FIGURE 2 | C. elegans lacking OGT-1 and OGA-1 activity show increased GaIN, GIcN, and Gal monosaccharides compared to N2 animals (B). Concentrations of monosaccharides galactosamaine (GalN) and glucosamine (GlcN) were measured by HPAEC as, immediately following their release, the $N$-acetyl sugars GalNAc and GlcNAc, respectively, undergo quantitative deacetylation. No statistically different changes were observed for the composition of PNGase $A$ and $F$ sensitive glycans for O-GlcNAc cycling mutants when compared to N2 animals (A) Error bars represent SD of an experiment done in triplicate. ns: $P>0.05$; ${ }^{*} P<0.05 ;{ }^{*} P<0.005 ;{ }^{*}{ }^{*} P<0.0005$ compared to N2.

\section{PNGase RESISTANT MONOSACCHARIDES ARE INCREASED IN ogt-1 NULL ANIMALS}

UDP-GlcNAc is required for the production of a myriad of glycans, which suggests that changes in its concentration may affect glycan synthesis. Indeed, increased pools of UDP-GlcNAc have been correlated with the production of tri- and tetra-antennary $\mathrm{N}$ glycans in mammary carcinoma cells (19). To determine whether complex glycan synthesis was affected by deletion of the C. elegans $\mathrm{O}$-GlcNAc cycling enzymes, we assessed whole animal glycan composition. Briefly, isolated N-glycans were released by PNGase $A$ and F, pooled, purified, and hydrolyzed with TFA. The resulting monosaccharide concentrations were assessed by HPAEC-PAD. Although ogt-1 animals exhibit over a twofold increase in UDPHexNAc levels compared to N2 (Figure 1), the released monosaccharides from N2, ogt-1 (ok430), ogt-1 (tm1046), and oga-1 (ok1207) animals' N-glycans remain largely similar (Figure 2A). Specifically, the ratio of mannose to GlcNAc suggests N-glycans form normally in these mutants.

Glycan structures not cleaved by PNGase A and F include O-linked glycans, glycolipids, and glycopolymers. To determine whether the PNGase-insensitive glycans were altered in ogt-1 and oga-1 null animals, the residual glycoprotein pellets were treated with $2 \mathrm{M}$ TFA and the freed sugar pools were assessed. The ogt-1 and oga-1 null animals exhibited up to 3.7-fold higher GalNAc, GlcNAc, and galactose levels compared to N2 worms suggesting structural changes in glycan composition for PNGase-insensitive glycans when O-GlcNAc cycling is perturbed (Figure 2B).

\section{GENES ENCODING ENZYMES INVOLVED IN UDP-GICNAc SYNTHESIS AND METABOLISM ARE PERTURBED IN O-GICNAc CYCLING MUTANTS}

With nucleotide sugar pools and PNGase-insensitive glycan structures affected by the loss of ogt-1 and oga-1, we hypothesized that the transcription of genes encoding enzymes involved in nucleotide sugar production and metabolism would be affected. 


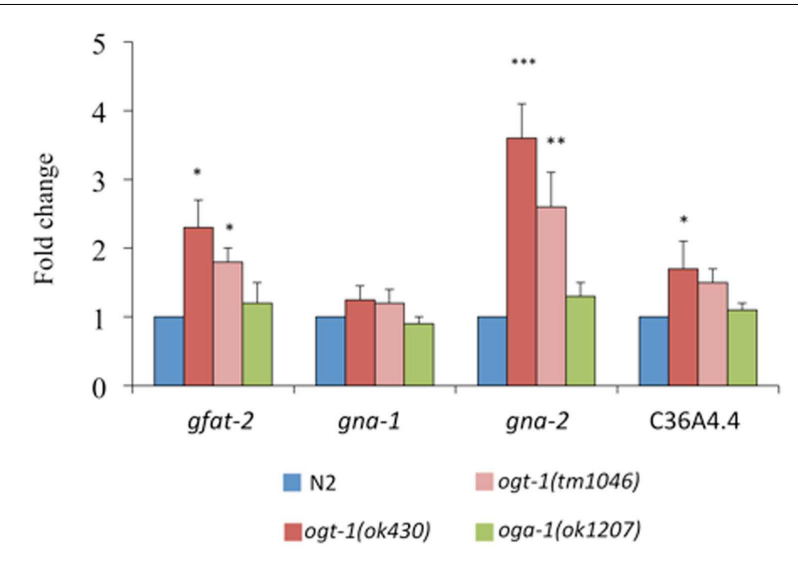

FIGURE 3 | Transcripts of genes encoding key HBP enzymes are elevated in ogt-1 null animals. Animals lacking OGT-1 activity show increased transcripts of gfat-2, gna-2, and C36A4.4 genes while animals lacking OGA-1 activity are not statistically different from N2. Error bars represent $\mathrm{SD}$ of an experiment done in triplicate. ns: $P>0.05 ;{ }^{*} P<0.05$; ${ }^{*} P<0.005 ;{ }^{*}{ }^{*} P<0.0005$ compared to N2.

To address this question, we examined the expression of key components of the HBP as well as trehalose and glycogen metabolism modules by qRT-PCR.

The following genes encode key enzymes in the HBP: gfat-2 (F22B3.4 encodes the HBP's rate-liming glucosamine:fructose-6phosphate aminotransferase - GFAT-2), gna-2 (a glucosamine-6phosphate $N$-acetyltransferase), and C36A4.4 (the putative UDPGlcNAc pyrophosphorylase orthologous to human UAP1) (20). Transcripts for gfat-2, gna-2, and C36A4.4 are all elevated in ogt1 (ok430) mutants while the transcript levels in oga-1 (ok1207) mutants remain unchanged (Figure 3). Elimination of OGT-1, an enzyme that utilizes a portion of the UDP-GlcNAc pool, affects the transcription of genes required for the synthesis of the same nucleotide sugar. This is not surprising as the HBP is exquisitely sensitive to nucleotide sugar concentrations and we suggest that gene transcription changes may be due to changes in feedback inhibition within the HBP. It is possible that there are no changes in HBP gene transcription for animals lacking OGA-1 activity as OGT-1 can still actively use UDP-GlcNAc.

Our previous work identified that total amounts of glycogen and trehalose, two important forms of energy storage, which can be enzymatically broken down to glucose, were increased in ogt1 and oga- 1 null animals (9). Here, we find that in comparison to N2 animals, ogt-1 null animals exhibit increased transcription for enzymes involved in trehalose and glycogen metabolism (tre1, tre-2, tps-2, and $g s y$-1) (Figure 4A). Interestingly, while ogt-1 (ok430) animals exhibit nearly twofold changes for all four genes, the oga-1 (ok1207) animals exhibited no statistical changes from $\mathrm{N} 2$ suggesting that changes in the ability of the animal to add and remove the O-GlcNAc modification have different biological consequences.

Triglyceride levels are significantly altered when OGT activity is perturbed in mice and C. elegans $(9,10,21)$. To better understand our data showing 40-70\% decreases in triglyceride levels in O-GlcNAc cycling mutants $(9,10)$, we assessed the level of

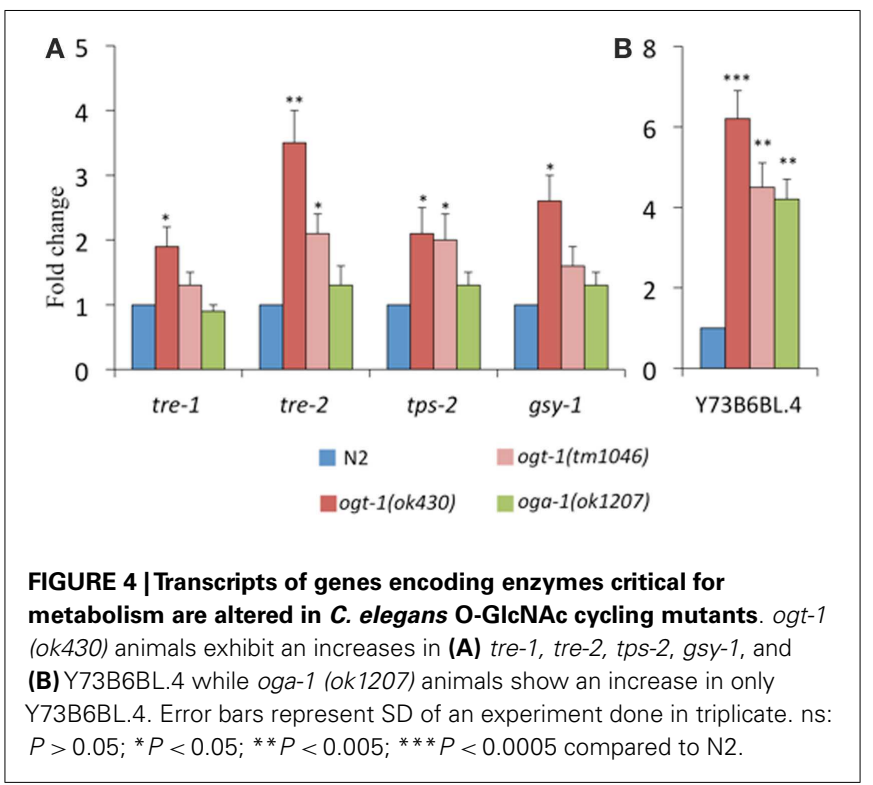

transcription of Y73B6BL.4, a gene encoding a phospholipase (22). Indeed, this phospholipase increased over fourfold for both ogt-1 alleles and the oga-1 (ok1207) animals (Figure 4B).

\section{DISCUSSION}

Cellular signaling depends on a series of protein posttranslational modifications working in concert to define protein localization, enzyme activity, and recognition events. Modifications ranging from glycosylation to phosphorylation are among the most widely recognized and defined posttranslational modifications with protein glycosylation being the most heterogeneous. The HBP produces a nutrient-sensitive nucleotide sugar, UDP-GlcNAc, that is utilized by glycosyltransferases in endo-membrane organelles (Golgi and endoplasmic reticulum) (23) as well as enzymes in the nucleus and cytoplasm (Figure 5). Optimal levels of UDPGlcNAc are required for maintenance of cellular homeostasis in mammals with profound consequences including embryonic lethality resulting from loss of the nucleotide sugar synthesis ( 7 , 24). The importance of this nucleotide sugar is evolutionarily conserved as loss or knockdown of enzymes required for synthesis of UDP-GlcNAc yields phenotypes including lethality in C. elegans (25-29).

Endo-membrane enzymes utilize UDP-GlcNAc to initiate Nglycosylation and further glycan branching (30). A portion of the UDP-GlcNAc pool is also used by OGT to glycosylate a myriad of nucleocytoplasmic targets ultimately influencing proteins' localization, activity, and/or folding [see Ref. $(8,31)$ and references therein]. Perturbation of O-GlcNAc cycling yields a wide range of biological consequences; indeed, OGT and OGA are essential for viability in mice and other higher eukaryotes $(11,32)$. In viable $C$. elegans animals with loss-of-function of ogt-1 and oga-1 genes, we note altered carbohydrate and lipid metabolism as well as severely deregulated insulin signaling $(9,10,33)$. Furthermore, we found that C. elegans animals with altered O-GlcNAc cycling have striking changes in fertility and reproductive timing during glucose stress suggesting that O-GlcNAc acts as a buffer to sense glucose 


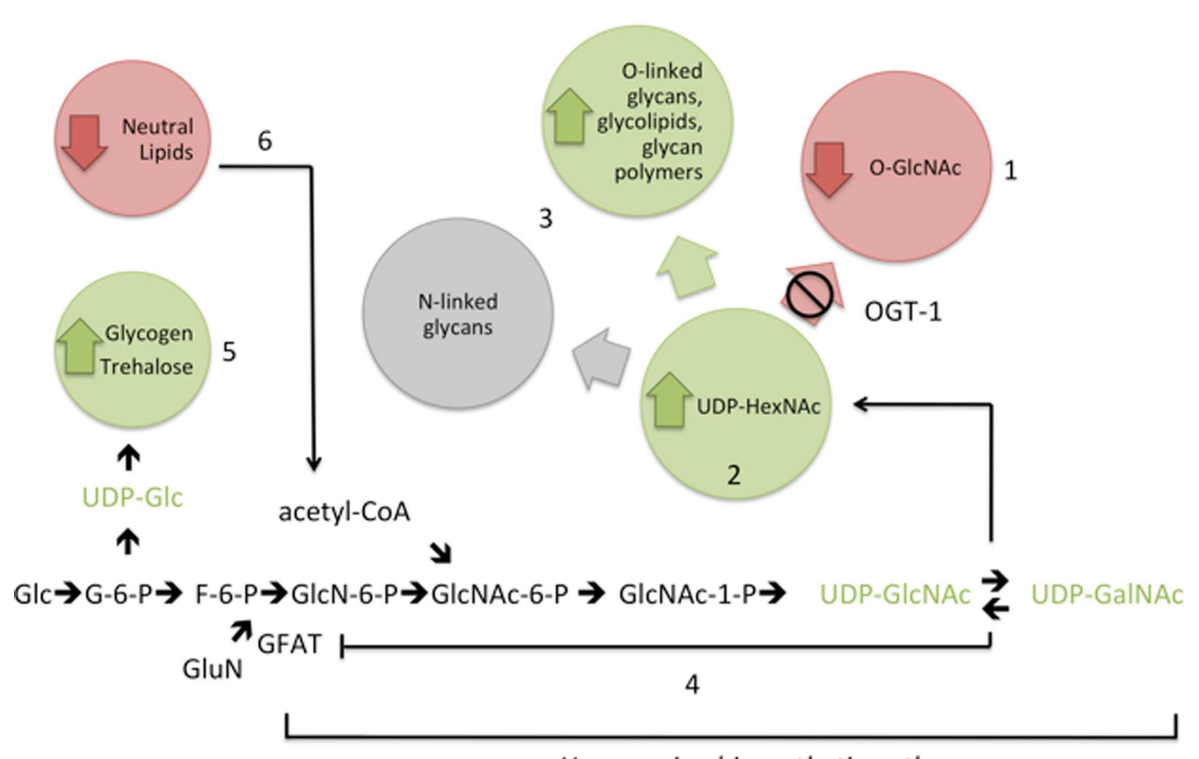

Hexosamine biosynthetic pathway

FIGURE 5 | Interference with the addition of O-GIcNAc (1) results in elevation of UDP-GIcNAc and UDP-GaINAc (UDP-HexNAc) levels (2)

Compensatory metabolic changes include increased flux into some complex glycans (3). Feedback inhibition of GFAT (4) is insufficient to fully normalize UDP-HexNAc levels in the absence of OGT-1. In addition, with increased nucleotide sugar pools, there are changes in upstream complex sugar levels (5) and decreased macronutrient storage (6). These changes in cellular nucleotide sugar pools are associated with altered transcriptional regulation of genes encoding hexosamine biosynthetic pathway and macronutrient storage enzymes (see text). Metabolite levels are increased (green), decreased (red), remain the same (gray), or were untested (black). Glc, glucose; G-6-P, glucose-6-phosphate; F-6-P, fructose-6-phosphate; GluN, glutamine; GIcN-6-P, glucosamine-6-phosphate; GlcNAc-6-P, N-acetylglucosamine-6-P; GIcNAc-1-P, $\mathrm{N}$-acetylglucosamine-1-phosphate; acetyl-CoA, acetyl coenzyme A. availability (34). The work herein details the way in which perturbations in O-GlcNAc cycling influences changes in overall $C$. elegans carbohydrate composition as well as the transcription of key metabolic enzymes.

Our first efforts focused on identifying the ways in which changes to O-GlcNAc cycling affected nucleotide sugar concentrations and overall cellular carbohydrate structure. We noted that UDP-Glc and UDP-HexNAc (a combination of UDP-GlcNAc and UDP-GalNAc) were elevated 1.5- to 3 -fold in the ogt-1 and oga1 animals (Figure 1). Importantly, other nucleotide sugars such as GDP-mannose were not altered suggesting specific changes to the pool of activated sugars directly downstream of glucose metabolism. The elevation of UDP-Glc and UDP-HexNAc in both O-GlcNAc cycling mutants intrigued us. It is clear that in ogt-1 loss-of-function animals, the UDP-GlcNAc pools would be elevated: the animals no longer utilize the activated nucleotide sugar to produce the O-GlcNAc PTM. Conversely, in animals that lack OGA-1 activity, while OGT-1 remains capable of utilizing UDPGlcNAc to glycosylate protein substrates, the cycling of the PTM is likely altered and may influence the turnover of the nucleotide sugar pool. We speculate that this may explain the modest changes in nucleotide sugar concentrations and alterations in PNGAseinsensitive glycans but yield milder effects on the transcription of HBP members.

We hypothesized that among the consequences for even modest changes in activated nucleotide sugar pools, animals lacking ogt-1 and oga-1 would exhibit changes in N-glycan structures. Indeed, $\mathrm{N}$-glycan structures have been shown to be ultrasensitive to UDP-GlcNAc concentration (19). We were, thus, surprised to find that $\mathrm{N}$-glycan monosaccharide composition in both ogt-1 and oga-1 animals was indistinguishable from N2 animals (Figure 2A). These data suggest that the C. elegans mutants lacking O-GlcNAc cycling to have no global defect in constructing glycans sensitive to PNGase $\mathrm{F}$ and $\mathrm{A}$. Interestingly, we found that glycans resistant to PNGase A or F (heterogeneous O-linked glycans, glycolipids, and glycan polymers) exhibited marked increases in galactose, GalNAc, and GlcNAc in both ogt-1 and oga-1 animals (Figure 2B). Recent work catalogs 14 types of mucin type O-linked glycans in the C. elegans WT strain with changes to O-linked glycans in another glycosyltransferase mutant (35). Future work will define the structural changes found in ogt-1 and oga-1 null animals' PNGase resistant glycans. The compiled data suggest that the inability to add and remove O-GlcNAc on nuclear and cytoplasmic targets has farreaching cellular affects affecting global glycosylation. These effects are likely to be pleiotropic and result from changes in transcription, metabolic flux, signaling, or direct enzyme activation. Among the reasons for these changes could be both direct and indirect perturbation of enzymes required for nucleotide sugar synthesis or the speed at which glycosylated proteins traffic through the secretory pathway (36).

With evidence suggesting that O-GlcNAc cycling is a key player in sensing cell nutrient status from our work and the work of others [see Ref. $(31,37)$ and references therein], we next hypothesized that the transcription of critical players in the HBP would be altered in the ogt- 1 and oga- 1 animals. The first, and rate limiting, enzyme of the HBP is GFAT, which is responsible for the conversion of 
fructose-6-phosphate to glucosamine-6-phosphate (Figure 5). To note, the activity of GFAT is modulated by UDP-GlcNAc itself through a feedback mechanism to reduce HBP flux (38). Two glucosamine-6-phosphate $N$-acetyltransferases (gna-1 and gna2) are required for an intermediary step in the HBP, and C36A4.4 is the presumptive pyrophosphorylase required for the last step in the synthesis of UDP-GlcNAc. We examined the expression levels for gfat-2, gna-1, gna-2, and C36A4.4 and noted that only in ogt-1 mutants were there statistically significant changes in transcription for three of the four transcripts (Figure 3). Although feedback inhibition should occur in ogt-1 animals due to increased UDPHexNAc levels, it is insufficient to normalize the nucleotide sugar pool with increased $g f a t-2$ transcription. Moreover, these findings were surprising as PNGase-insensitive glycans were altered in both mutants suggesting a complex interplay between nutrient flux and appropriate substrate O-GlcNAc modification. (37).

Given that the O-GlcNAc cycling mutants exhibit variations in activated nucleotide sugar pools, changes in glycosylation (Figures 1 and 2), and increased glycogen and trehalose storage (9), we next assessed the levels of transcription for genes encoding enzymes involved in trehalose and glycogen metabolism. C. elegans encodes four putative glycoside hydrolases - enzymes responsible for catalyzing the conversion of trehalose to glucose - including tre- 1 and tre-2. tps- 2 encodes for one of two enzymes responsible for trehalose-6-phosphate synthesis and $g s y-1$ is ortholog to the human glycogen synthase 1 . All four of these transcripts were found to be elevated in ogt-1 mutants while the levels remain unchanged in oga-1 animals (Figure 4). These findings are consistent with our previous reports suggesting major changes in trehalose and glycogen metabolism upon genetic interference with $\mathrm{O}-\mathrm{GlcNAc}$ cycling. The present findings suggest that these changes are associated with increased metabolic flux to produce UDP-Glc and with transcriptional changes in the transcripts encoding the relevant enzymes mediating interconversion.

Triglyceride levels have been shown to correlate with perturbations in O-GlcNAc cycling in both mice and C. elegans $(9,10,21)$ and free fatty acids, usually derived from triglycerides or phospholipids, are known to be potent HBP modulators (39). Our previous work demonstrated that triglyceride levels are decreased by $70 \%$ in ogt 1 and $40 \%$ in oga- 1 compared to N2 (9). These data suggest that either the production of triglycerides was hampered or their hydrolysis was increased. To test whether triglycerides are catabolized more rapidly in the O-GlcNAc cycling mutants, we assessed the transcriptional expression of Y73B6BL.4, a gene encoding for a phospholipase. We noted a significant increase in transcription for Y73B6BL. 4 in both O-GlcNAc cycling mutants supporting that the decrease in triglyceride levels for both ogt-1 and oga-1 animals is likely associated with increased hydrolysis.

Perturbations of OGA activity in cell culture (40-42), loss of OGA-1 activity in C. elegans $(9,10)$, and OGT overexpression in mouse liver or fat promotes insulin resistance (43). Furthermore, loss of OGT-1 yields insulin sensitivity in C. elegans and altered lipid and carbohydrate metabolism $(9,10)$. Together, these data support a strong role for O-GlcNAc in insulin signaling metabolism maintenance. Our results reveal that loss of ogt-1 and oga-1 changes the nucleotide sugar pools and the production of PNGase-insensitive glycans. These changes along with altered transcriptional expression of genes encoding key HBP and metabolic enzymes in ogt-1 null animals suggest that the addition of O-GlcNAc to appropriate target proteins is critical for appropriate HBP flux. Additional roles of OGT, including its non-catalytic role in protein-protein interactions (44), could also influence this signaling paradigm. We propose that with O-GlcNAc cycling profoundly affecting the HBP, C. elegans is an excellent model to studying metabolic changes associated with insulin signaling in viable ogt-1 and oga-1 null alleles (Figure 5). Using C. elegans ogt1 and $o g a-1$ animals, we will be able to further define the molecular details of the HBP's role in insulin resistance.

\section{ACKNOWLEDGMENTS}

The authors wish to thank members of the Krause and Hanover labs for their helpful comments and discussion.

\section{REFERENCES}

1. Rexach JE, Clark PM, Hsieh-Wilson LC. Chemical approaches to understanding O-GlcNAc glycosylation in the brain. Nat Chem Biol (2008) 4:97-106 doi: $10.1038 /$ nchembio. 68

2. Ma J, Hart GW. O-GlcNAc profiling: from proteins to proteomes. Clin Proteomics (2014) 11:8. doi:10.1186/1559-0275-11-8

3. Hanover JA. Glycan-dependent signaling: O-linked $\mathrm{N}$-acetylglucosamine. FASEB J (2001) 15:1865-76. doi:10.1096/fj.01-0094rev

4. Roquemore EP, Chevrier MR, Cotter RJ, Hart GW. Dynamic O-GlcNAcylation of the small heat shock protein alpha B-crystallin. Biochemistry (1996) 35:3578-86 doi:10.1021/bi951918j

5. Love DC, Hanover JA. The hexosamine signaling pathway: deciphering the "OGlcNAc code". Sci STKE (2005) 2005:re13. doi:10.1126/stke.3122005re13

6. Teo CF, Wollaston-Hayden EE, Wells L. Hexosamine flux, the O-GlcNAc modification, and the development of insulin resistance in adipocytes. Mol Cell Endocrinol (2010) 318:44-53. doi:10.1016/j.mce.2009.09.022

7. Greig KT, Antonchuk J, Metcalf D, Morgan PO, Krebs DL, Zhang JG, et al. Agm1/Pgm3-mediated sugar nucleotide synthesis is essential for hematopoiesis and development. Mol Cell Biol (2007) 27:5849-59. doi:10.1128/MCB.00802-07

8. Bond MR, Hanover JA. O-GlcNAc cycling: a link between metabolism and chronic disease. Annu Rev Nutr (2013) 33:205-29. doi:10.1146/annurev-nutr071812-161240

9. Forsythe ME, Love DC, Lazarus BD, Kim EJ, Prinz WA, Ashwell G, et al. Caenorhabditis elegans ortholog of a diabetes susceptibility locus: oga-1 (OGlcNAcase) knockout impacts O-GlcNAc cycling, metabolism, and dauer. Proc Natl Acad Sci U S A (2006) 103:11952-7. doi:10.1073/pnas.0601931103

10. Hanover JA, Forsythe ME, Hennessey PT, Brodigan TM, Love DC, Ashwell G, et al. A Caenorhabditis elegans model of insulin resistance: altered macronutrient storage and dauer formation in an OGT-1 knockout. Proc Natl Acad Sci U S A (2005) 102:11266-71. doi:10.1073/pnas.0408771102

11. Shafi R, Iyer SP, Ellies LG, O'Donnell N, Marek KW, Chui D, et al. The OGlcNAc transferase gene resides on the $\mathrm{X}$ chromosome and is essential for embryonic stem cell viability and mouse ontogeny. Proc Natl Acad Sci U S A (2000) 97:5735-9. doi:10.1073/pnas.100471497

12. Brenner S. The genetics of Caenorhabditis elegans. Genetics (1974) 77:71-94.

13. Lewis JA, Fleming JT. Basic culture methods. Methods Cell Biol (1995) 48:3-29. doi:10.1016/S0091-679X(08)61381-3

14. Suriano R, Ghosh SK, Ashok BT, Mittelman A, Chen Y, Banerjee A, et al. Differences in glycosylation patterns of heat shock protein, gp96: implications for prostate cancer prevention. Cancer Res (2005) 65:6466-75. doi:10.1158/00085472.CAN-04-4639

15. Cipollo JF, Costello CE, Hirschberg CB. The fine structure of Caenorhabditis elegans N-glycans. J Biol Chem (2002) 277:49143-57. doi:10.1074/jbc.M208020200

16. Cipollo JF, Awad AM, Costello CE, Hirschberg CB. N-Glycans of Caenorhabditis elegans are specific to developmental stages. J Biol Chem (2005) 280:26063-72. doi:10.1074/jbc.M503828200

17. Banerjee A, Wang R, Supernavage SL, Ghosh SK, Parker J, Ganesh NF, et al. Implications of phase variation of a gene (pgtA) encoding a pilin galactosyl transferase in gonococcal pathogenesis. J Exp Med (2002) 196:147-62. doi:10.1084/jem.20012022 
18. Fukushige T, Brodigan TM, Schriefer LA, Waterston RH, Krause M. Defining the transcriptional redundancy of early bodywall muscle development in C. elegans: evidence for a unified theory of animal muscle development. Genes Dev (2006) 20:3395-406. doi:10.1101/gad.1481706

19. Lau KS, Partridge EA, Grigorian A, Silvescu CI, Reinhold VN, Demetriou M, et al. Complex N-glycan number and degree of branching cooperate to regulate cell proliferation and differentiation. Cell (2007) 129:123-34. doi:10.1016/j.cell. 2007.01.049

20. Shaye DD, Greenwald I. OrthoList: a compendium of C. elegans genes with human orthologs. PLoS One (2011) 6:e20085. doi:10.1371/journal.pone. 0020085

21. Yang X, Ongusaha PP, Miles PD, Havstad JC, Zhang F, So WV, et al. Phosphoinositide signalling links O-GlcNAc transferase to insulin resistance. Nature (2008) 451:964-9. doi:10.1038/nature06668

22. Morrison K, Witte K, Mayers JR, Schuh AL, Audhya A. Roles of acidic phospholipids and nucleotides in regulating membrane binding and activity of a calciumindependent phospholipase A2 isoform. J Biol Chem (2012) 287:38824-34. doi:10.1074/jbc.M112.391508

23. Hirschberg CB, Robbins PW, Abeijon C. Transporters of nucleotide sugars, ATP, and nucleotide sulfate in the endoplasmic reticulum and Golgi apparatus. Annu Rev Biochem (1998) 67:49-69. doi:10.1146/annurev.biochem.67.1.49

24. Boehmelt G, Wakeham A, Elia A, Sasaki T, Plyte S, Potter J, et al. Decreased UDPGlcNAc levels abrogate proliferation control in EMeg32-deficient cells. EMBO J (2000) 19:5092-104. doi:10.1093/emboj/19.19.5092

25. Johnston WL, Krizus A, Dennis JW. The eggshell is required for meiotic fidelity, polar-body extrusion and polarization of the C. elegans embryo. BMC Biol (2006) 4:35. doi:10.1186/1741-7007-4-35

26. Kamath RS, Fraser AG, Dong Y, Poulin G, Durbin R, Gotta M, et al. Systematic functional analysis of the Caenorhabditis elegans genome using RNAi. Nature (2003) 421:231-7. doi:10.1038/nature01278

27. Piano F, Schetter AJ, Morton DG, Gunsalus KC, Reinke V, Kim SK, et al. Gene clustering based on RNAi phenotypes of ovary-enriched genes in C. elegans. Curr Biol (2002) 12:1959-64. doi:10.1016/S0960-9822(02)01301-5

28. Rual JF, Ceron J, Koreth J, Hao T, Nicot AS, Hirozane-Kishikawa T, et al. Toward improving Caenorhabditis elegans phenome mapping with an ORFeome-based RNAi library. Genome Res (2004) 14:2162-8. doi:10.1101/gr.2505604

29. Sönnichsen B, Koski LB, Walsh A, Marschall P, Neumann B, Brehm M, et al. Full-genome RNAi profiling of early embryogenesis in Caenorhabditis elegans. Nature (2005) 434:462-9. doi:10.1038/nature03353

30. Lowe JB, Marth JD. A genetic approach to mammalian glycan function. Annu Rev Biochem (2003) 72:643-91. doi:10.1146/annurev.biochem.72.121801. 161809

31. Zachara NE, Hart GW. O-GlcNAc a sensor of cellular state: the role of nucleocytoplasmic glycosylation in modulating cellular function in response to nutrition and stress. Biochim Biophys Acta (2004) 1673:13-28. doi:10.1016/j.bbagen.2004. 03.016

32. Yang YR, Song M, Lee H, Jeon Y, Choi EJ, Jang HJ, et al. O-GlcNAcase is essential for embryonic development and maintenance of genomic stability. Aging Cell (2012) 11:439-48. doi:10.1111/j.1474-9726.2012.00801.x

33. Love DC, Ghosh S, Mondoux MA, Fukushige T, Wang P, Wilson MA, et al. Dynamic O-GlcNAc cycling at promoters of Caenorhabditis elegans genes regulating longevity, stress, and immunity. Proc Natl Acad Sci U S A (2010) 107:7413-8. doi:10.1073/pnas.0911857107
34. Mondoux MA, Love DC, Ghosh SK, Fukushige T, Bond M, Weerasinghe GR, et al. O-linked-N-acetylglucosamine cycling and insulin signaling are required for the glucose stress response in Caenorhabditis elegans. Genetics (2011) 188:369-82. doi:10.1534/genetics.111.126490

35. Parsons LM, Mizanur RM, Jankowska E, Hodgkin J, O’Rourke D, Stroud D, et al. Caenorhabditis elegans bacterial pathogen resistant bus-4 mutants produce altered mucins. PLoS One (2014) 9:e107250. doi:10.1371/journal.pone.0107250

36. Wellen KE, Thompson CB. A two-way street: reciprocal regulation of metabolism and signalling. Nat Rev Mol Cell Biol (2012) 13:270-6. doi:10.1038/ nrm3305

37. Hanover JA, Krause MW, Love DC. The hexosamine signaling pathway: OGlcNAc cycling in feast or famine. Biochim Biophys Acta (2010) 1800:80-95. doi:10.1016/j.bbagen.2009.07.017

38. Kornfeld S, Kornfeld R, Neufeld EF, O’Brien PJ. The feedback control of sugar nucleotide biosynthesis in liver. Proc Natl Acad Sci U S A (1964) 52:371-9. doi:10.1073/pnas.52.2.371

39. Wang J, Liu R, Hawkins M, Barzilai N, Rossetti L. A nutrient-sensing pathway regulates leptin gene expression in muscle and fat. Nature (1998) 393:684-8. doi: $10.1038 / 31474$

40. Arias EB, Kim J, Cartee GD. Prolonged incubation in PUGNAc results in increased protein O-Linked glycosylation and insulin resistance in rat skeletal muscle. Diabetes (2004) 53:921-30. doi:10.2337/diabetes.53.4.921

41. Park SY, Ryu J, Lee W. O-GlcNAc modification on IRS-1 and Akt2 by PUGNAc inhibits their phosphorylation and induces insulin resistance in rat primary adipocytes. Exp Mol Med (2005) 37:220-9. doi:10.1038/emm.2005.30

42. Vosseller K, Wells L, Lane MD, Hart GW. Elevated nucleocytoplasmic glycosylation by $\mathrm{O}-\mathrm{GlcNAc}$ results in insulin resistance associated with defects in Akt activation in 3T3-L1 adipocytes. Proc Natl Acad Sci U S A (2002) 99:5313-8. doi:10.1073/pnas.072072399

43. McClain DA, Lubas WA, Cooksey RC, Hazel M, Parker GJ, Love DC, et al. Altered glycan-dependent signaling induces insulin resistance and hyperleptinemia. Proc Natl Acad Sci U S A (2002) 99:10695-9. doi:10.1073/pnas.152346899

44. Iyer SP, Hart GW. Roles of the tetratricopeptide repeat domain in O-GlcNAc transferase targeting and protein substrate specificity. J Biol Chem (2003) 278:24608-16. doi:10.1074/jbc.M300036200

Conflict of Interest Statement: The authors declare that the research was conducted in the absence of any commercial or financial relationships that could be construed as a potential conflict of interest.

Received: 03 September 2014; accepted: 10 November 2014; published online: 24 November 2014.

Citation: Ghosh SK, Bond MR, Love DC, Ashwell GG, Krause MW and Hanover JA (2014) Disruption of O-GlcNAc cycling in C. elegans perturbs nucleotide sugar pools and complex glycans. Front. Endocrinol. 5:197. doi: 10.3389/fendo.2014.00197

This article was submitted to Molecular and Structural Endocrinology, a section of the journal Frontiers in Endocrinology.

Copyright (c) 2014 Ghosh, Bond, Love, Ashwell, Krause and Hanover. This is an openaccess article distributed under the terms of the Creative Commons Attribution License (CC BY). The use, distribution or reproduction in other forums is permitted, provided the original author(s) or licensor are credited and that the original publication in this journal is cited, in accordance with accepted academic practice. No use, distribution or reproduction is permitted which does not comply with these terms. 